\title{
LA RESISTENCIA ANTE EL BIOPODER MOLECULAR
}

\author{
Resistance to molecular biopower
}

\author{
RICARDO CAMARGO \\ Universidad de Chile (Chile) \\ rcamargo@derecho.uchile.cl \\ NICOLÁS RIED \\ Universidad de Chile (Chile) \\ nicolasried@gmail.com
}

\section{Resumen}

En Towards a genealogy of pharmaceutical practice (Camargo y Ried, 2016), sostuvimos que la práctica farmacológica daba lugar a un nuevo tipo de biopoder (“biopoder molecular”), con tecnologías y racionalidades propias, que lo distinguían del biopoder que había reconocido Michel Foucault en su trabajo tardío. Pero también sostuvimos que su contracara era la generación de nuevas formas de resistencia. En este artículo presentaremos dos modelos específicos de resistencias producidos por el dispositivo del poder farmacológico: uno, que tiene como caso básico el de los movimientos proanorexia, que operan resignificando la categoría misma de la patología "anoréxica" a base del fármaco Xenical; otro, que tiene referencia en la experiencia relatada por Beatriz Preciado en su libro Testo yonqui (2008) y que actúa superando los límites legales, médicos y morales, para arrojarse a la producción de una nueva identidad jamás antes trazada al consumir dosis no permitidas de testosterona en gel.

Palabras clave: Resistencia; Proana; Testosterona; Xenical; Biopoder.

\section{Abstract}

In a previous work (Camargo \& Ried, 2016), we have argued that pharmacological practice has given rise to a new type of biopower ("molecular biopower"), with specific technologies and rationalities, distinguishing it from the biopower that Michel Foucault recognised in his later work. However, we also argued that another effect has been the creation of new forms of resistance. In this article, we present two specific models of resistance created by the apparatus of pharmacological power: one, inspired by pro-anorexia movements, which operates by resignifying the pathological categorization of anorexia using the drug Xenical; another, based on the experience outlined by Beatriz Preciado in her 2008 book Testo Junkie, which operates by overcoming legal, medical and moral limits to create a ground-breaking new identity by consuming prohibited doses of gel testosterone.

Key words: Resistance; Pro-ana; Testosterone; Xenical; Biopower. 


\section{RESISTENCIA: UNA BREVE GENEALOGÍA DE LA DISCUSIÓN}

Desde la definición que nos diera Michel Foucault del poder, como aquella situación estratégica compleja cuyo anverso es la resistencia (Foucault, 2008, pp. 89 y 91) ${ }^{1}$, los intentos por definir a esta última han proliferado exponencialmente. Se asocia intrínsecamente la noción de poder con la de resistencia, implicando que la tarea política principal ya no es solamente identificar y develar las tecnologías y racionalidades que constituyen el poder, sino que además es relevante pensar en las prácticas de resistencia de manera propositiva (Rose y Miller, 2008). Es en esos términos que la noción de resistencia se ha asociado a la de lucha, ya que en la obra de Foucault la cuestión política no se centra tanto en el plano de la denuncia del poder, como sí en las estrategias que se emplean para desbaratarlo: la resistencia se presenta más bien como una lucha localizada, con tácticas específicas y no un esquema general de oposición a una estructura o sistema determinado. Siguiendo la lectura del especialista Edgardo Castro (2004), podemos reconocer tres formas que presenta el poder en la obra de Foucault: dominación, explotación y sujeción. Ante estas formas del poder, se muestran tres correspondientes maneras de luchar (p. 263). La dominación ejerce el poder por medio de las diversas racionalidades y tecnologías que clasifican a los individuos en una cierta etnia, en una raza específica, en una religión determinada, regulando sus prácticas y sometiéndolos a prohibiciones normativas cuyo quebrantamiento trae aparejado un castigo, característico de las sociedades feudales. La explotación, como ejercicio del poder, es el modo en que se aliena a los individuos de aquello que producen, que en la fórmula marxista se representa como la separación entre trabajo y plusvalía. Foucault reconoce que la dominación y la explotación son expresiones históricamente situadas del poder, cuya manifestación produce ciertas formas específicas de resistencia. Sin embargo, la forma contemporánea de expresión del poder, dirá Foucault, es la sujeción, que en este contexto significa el modo en que el poder produce una sumisión de la subjetividad mediante ciertos dispositivos y tecnologías 2 . Que sean modulaciones

\footnotetext{
${ }^{1}$ Literalmente, Michel Foucault escribe: "El poder no es una institución, y no es una estructura, no es cierta potencia de la que algunos estarían dotados: es el nombre que se presta a una situación estratégica compleja [...] Donde hay poder hay resistencia, y no obstante (precisamente por esto), esta nunca está en posición de exterioridad respecto del poder". Para una noción del poder como facilidad: Heller, Kevin Jon. "Power, subjectivation and resistance in Foucault" (1996, pp. 78-110).

2 Acerca del uso de este término existe anfibología: el término en inglés, "subjection", puede significar tanto "sujeción" como "sometimiento"; mientras que el término en francés "assujetissement", utilizado por Michel Foucault, puede ser traducido como "sujeción" o "subjetivación”. Es por ello que aquí el concepto de sujeción se presenta con esa doble significación: por una parte es el verse sujeta la identidad a ciertas tecnologías y dispositivos de poder que la regulan, y por otra es la acción de someterse a un proceso de subjetivación específico. Aquí utilizamos la noción de "sujeción", porque el término subjetivación ha adquirido un significado específico emancipatorio, como práctica de subversión de la identidad ante el sometimiento de ciertos dispositivos, como lo hacen las lecturas de Rancière (2006) y Ried (2016, pp. 87-96).
}

10 | Alpha No 50 (Julio 2020) PÁGS. 9-20. ISSN 07 16-4254 
históricas, no significa que su presencia como expresión del poder sea excluyente, por el contrario, la sujeción existiría coetáneamente con la dominación y la explotación. A la sujeción, Foucault opone las técnicas del cuidado de sí como tipo de resistencia que adquiere la forma de desujeción. Esta concepción de la resistencia, como anverso del poder, se presenta de diversas maneras, con distintos nombres, pero siempre como una práctica doble de producción. En tal sentido, tanto el poder como la resistencia crean, producen y construyen subjetividad o desubjetividades. La resistencia en su modalidad de desujeción no sería por tanto una simple negación o prohibición, ni tampoco llana censura o silencio (Ried, 2016, p. 81).

En una línea similar, Brent L. Pickett ha sostenido que el concepto de resistencia en Foucault presenta tres fases, las que a su vez constituyen un continuo: un primer período que se enfoca en la diferencia, una segunda etapa que se expresa en la revolución y un tercer momento que da lugar a la noción de resistencia como tal. En el primer período, el poder se presenta como una serie de "límites" que marcan una diferencia entre dos elementos aparentemente dicotómicos: racional/irracional, bien/mal, normal/anormal. La resistencia se presenta como afirmación de la negación, como oposición al límite que separa las dos caras de la aparente dicotomía. Historia de la locura (1961) y Locura y civilización (1967) son obras representativas de este período. En un segundo momento, el análisis se centra en la manera en que ciertas instituciones derramarían el poder regulando prácticas y reproduciendo la ideología normalizadora en una comunidad. La resistencia se tornaría revolucionaria, al combatir las instituciones en su totalidad: la escuela, la clínica, la prisión son instituciones clásicas del análisis que Foucault realiza en este período. El poder psiquiátrico (1973-1974) y Vigilar y castigar (1975) son los textos en los que Foucault vierte su reflexión acerca de este asunto. En tercer lugar se despliega la noción de prácticas de resistencia ante un poder difuso y complejo. Aquí es relevante el descubrimiento de los conceptos poder y resistencia, que permiten un análisis dinámico y específico de las formas en que el poder se manifiesta. Foucault trabaja conceptos como los de cuidado de sí y tecnologías del yo como formas de oponerse al poder que produce subjetividades. Los últimos trabajos de Foucault pertenecen a este período, siendo de principal relevancia Historia de la sexualidad (1976-1984) y sus últimas clases del Colegio de Francia.

El orden cronológico que ofrece Pickett (1996) en torno a la resistencia, coincide con el que Castro (2004) nos entrega de la lucha. Cuando Foucault analiza formas de manifestación del poder, debemos comprender que también muestra los modos en que se expresa la resistencia, así: ante la dominación es la diferencia, ante la explotación es la revolución y ante la sujeción es la resistencia.

Sostenemos, sin embargo, que la noción de resistencia es particularmente útil en el lenguaje político cuando es comprendida en su denotación específica de desujeción. La resistencia como desujeción nos entrega respuestas a uno de los puntos más cuestionados en el esquema foucaultiano: ¿por qué luchar, por qué resistir? $\mathrm{Si}$ 
consideramos estas preguntas desde la perspectiva de la desujeción, entenderemos que el punto central abogado por Foucault en la resistencia no es tanto reaccionar frente a algo sino construirse a sí mismo en esa reacción. En tal sentido, la resistencia sería una virtud característica de la vida nueva, ya que existimos en tanto hacemos algo original de nosotros mismos.

En esta misma línea Jocelyn A. Hollander y Rachel L. Einwohner (2004) han elaborado una sistematización de la batalla por el concepto de resistencia que tipifica los modos en que se la ha intentado comprender, a base de sus ideas nucleares y a dos criterios de diferenciación (pp. 533-554). Las autoras sostienen que los elementos comunes en todas las concepciones de la resistencia son los de acción y oposición, es decir, que toda resistencia actúa de manera opuesta a una cierta situación de dominación, explotación o violencia. Por otra parte, un acto de resistencia requiere de cierta intención por parte de su autor/a y de un cierto grado de reconocimiento por la comunidad en la que ese acto opera. Estos dos últimos factores aluden cada uno a un criterio interno y otro externo de identificación de la resistencia. A partir de ellos, se identifican diversos tipos de resistencia, siendo de particular interés para este análisis el que Hollander y Einwohner denominan como "no resistencia", esto es: una acción de oposición sin intención ni criterios de reconocimiento, lo que supone un acto que carece de sentido y que no tiene un horizonte. Esto es interesante, pues una de las cuestiones que intentaremos resolver en este artículo es si ¿puede haber resistencia sin que haya horizonte de sentido? $\mathrm{O}$ en otras palabras, ¿toda resistencia debe tener intenciones, metas y criterios de reconocimiento ciertos?.

En efecto, lo que parecen dar por establecido las nociones de resistencia presentadas por Hollander y Einwohner es un cierto horizonte de sentido hacia el que se dirige la acción resistente. Sin embargo, al situarla en una relación profunda con la constitución de sí mismo, la cuestión deviene aleatoria: hacemos algo con nosotros, pero no sabemos necesariamente qué. Existe una intención de constitución propia, pero sin un mapa claro ${ }^{3}$.

Para exponer este problema, analizaremos a continuación dos casos específicos que denotan gráficamente la tensión entre circunscripción y apertura que habita la noción de resistencia como desujeción, y haremos mención a los problemas para lo político a que dicha tensión reconduce.

El primer caso refiere a los movimientos proanorexia que buscarán resignificar su "patología" bregando por hacer de ella un "estilo de vida" digno de ser reivindicado como tal (O'Connor y Van Esterik, 2008). El segundo, es el que se deriva de la obra de Beatriz Preciado, en particular de su libro Testo yonqui (2014), en el que la desujeción aparecerá radicalmente abierta e indeterminada.

\footnotetext{
${ }^{3}$ Para una presentación del problema de la resistencia más allá del horizonte de sentido en la obra de Foucault véase Camargo (2010, pp. 315-332).

12 | Alpha № 50 (Julio 2020) PÁGs. 9-20. ISSN 07 16-4254
} 


\section{MOVIMIENTOS PRO-ANA: HACIA UNA RESISTENCIA POR RESIGNIFICACIÓN}

La anorexia ha sido comúnmente catalogada como una patología por las ciencias médicas. Hacia 1870 era denominada como anorexia nervosa, una condición psiquiátrica concebida como un desorden mental que afecta peligrosamente la masa corporal, especialmente de las adolescentes (Fox, Ward y O'Rourke, 205, p. 947). Al ser clasificada como una enfermedad, el tratamiento prescrito por los médicos combina habitualmente terapias psicológicas y consumo de medicamentos. En paralelo, sin embargo, se han desplegado desde las humanidades y las ciencias sociales esquemas analíticos que ven en el modelo patriarcal de percepción de la belleza el principal factor explicativo de la anoréxica. Las jóvenes tenderían a seguir ciertos patrones de delgadez, a riesgo de su salud, movidas por la exigencia de adecuarse a una estética estereotipada impuesta por una mirada machista de los cuerpos (p. 947).

El punto a tener en cuenta es que médicos y científicos sociales coinciden en tratar la anorexia como una patología, lo que alienta la constitución correlativa de un dispositivo de medicina correctiva y preventiva que busca la normalización de la estética y la salud corporal, principalmente de adolescentes. Para ello se prescribe un régimen alimenticio estándar que dicta qué y cuánto comer para sanarse. Legitimado desde el dispositivo médico, el régimen alimenticio ha sido rápidamente colonizado por lógicas comerciales y por el surgimiento de una industria de alimentación sana o light que estimula el cultivo de sujetos saludables. Se trata de una "biopolítica de la vida saludable", cuya caracterización y modalidades ha tenido abundante tratamiento en la literatura reciente. ${ }^{4}$ Menos documentadas, sin embargo, desde la perspectiva biopolítica, son las prácticas de resistencia que han comenzado a constituirse frente al régimen de vida saludable entendido como estándar de normalidad. Una de dichas prácticas son los movimientos proanorexia (o proana) que se visibilizan en foros de internet a comienzos de la década de 1990, y se potencian en la década del 2000. Son comunidades -básicamente de adolescentes, casi exclusivamente mujeres- que se constituyen en resistencia a la patologización de la anorexia nervosa. Se trata de una disputa doble. En primer lugar, en contra del diagnóstico como enfermedad de la anorexia proveído por la disciplina médica; y en segundo término, en contra de la estereotipación de la anorexia proveída por la perspectiva humanista o de las ciencias sociales, que la observa como un mero efecto de una opresión de género. Ante estas clasificaciones, las comunidades proana reivindican la decisión autónoma de asumirse como ana.

En un extenso trabajo de campo Richard O'Connor y Penny van Esterik (2008) muestran que para los movimientos proana, la anorexia lejos de ser considerada una

\footnotetext{
${ }^{4}$ Interesantes apreciaciones sobre este asunto en: Metzl y Kirkland, 2010; Nadesan, 2010; Karmy, 2014. AlPHA No 50 (Julio 2020) PÁGs. 9-20. ISSN 07 16-4254| 13
} 
anormalidad es más bien comprendida como un estilo de vida (p. 6). De esta manera, las comunidades proana luchan por desplazar la anorexia desde el ámbito de las ciencias (médicas y sociales) para situarla como parte de una discusión ética y estética (la ética y la estética de la existencia), en este caso, relativa al estatus de las prácticas alimenticias. Una ética-estética que no se satisface con parámetros estándares de belleza y que más bien apunta a criterios de virtud.

Esta orientación hacia la virtud, ubicaría a estas comunidades proana promoviendo prácticas de carácter ascético, que combinan el cultivo de la autodisciplina, una búsqueda de la perfección y una performance que despliega modelos de belleza no tradicional (O’Connor y Van Esterik, 2008, p. 9). Se trata de un virtuosismo que puede incluso alcanzar ribetes heroicos, como lo documenta Sigal Gooldin en su artículo Being anorexic:

The findings of this study suggest that anorexic women actively construct a 'heroic moral subjectivity' in which hunger plays a crucial role and in which everyday (mundane) practices acquire out-of-the-ordinary meanings (2008, p. 275) .

Del mismo modo, las prácticas de las comunidades proana se alejan también de la crítica feminista tradicional que sitúa a la anorexia como una mera manifestación de la alianza entre patriarcado y capitalismo, que haría del cuerpo femenino una mercancía sujeta a parámetros de consumo inhumanos. Más aún, la crítica feminista ha tendido a presentar a la anorexia como un fenómeno cultural que es mera consecuencia de la posición y el valor que las mujeres tienen en la sociedad, como un sujeto-objeto inferior dispuesto para el goce patriarcal (Malson). Sin embargo, el estudio de las comunidades proana ha permitido visualizar una posibilidad que permanece precluida en la lectura que difunde el feminismo tradicional, a saber: que la palabra de las "pacientes" anoréxicas tiene un valor propio, una autenticidad emanada del decidir vivir como ana - una posibilidad inquietante para el discurso normalizante, pero también para parte importante de la lectura feminista que no logra soportar que haya mujeres que se sientan cómodas viviendo en una estética que desprecian—(Hope, Tan, Stewart y Fitzpatrick, 2011).

¿Cómo se ha constituido esta práctica en la que las comunidades proana han pugnado por despatologizar la condición de anorexia? Las proana se organizan principalmente de manera virtual, mediante blogs y chats de internet que les posibilitan una comunicación expedita de sus propios testimonios y en especial de sus técnicas o tips para perder peso. Una de las experiencias más compartidas es el uso de medicamentos, en particular del Xenical u Orlistat normalmente indicado para controlar la baja excesiva de peso. El Xenical es un fármaco que comúnmente requiere ser recetado por un

\footnotetext{
5 "Los resultados de este estudio muestran que las mujeres anoréxicas construyen activamente una 'subjetividad moral heroica' en la cual el hambre juega un rol crucial y en la que prácticas diarias (mundanas) adquieren un significado fuera de lo normal" (Trad., mía).
}

14 | AlPHA No 50 (Julio 2020) PÁGs. 9-20. ISSN 07 16-4254 
facultativo médico, pero que sin embargo es de fácil acceso mediante su compra en internet. Lo interesante del uso del Xenical por parte de las comunidades proana es que su finalidad original (regular la baja de peso) es modificada e invertida, sirviendo ahora como técnica que permite hacer sustentable la condición anoréxica de aquellas/os que deciden adoptarla, controlando los riesgos extremos e inmediatos para la vida. El punto a tener en cuenta no es si esta subversión del saber científico es correcta, esto es, si logra su objetivo (hacer sustentable la vida anoréxica), sino que en su despliegue se produce performativamente un tipo de vida nueva. Como han documentado N. J. Fox, K. J. Ward y A. J. O'Rourke (2005) esta inversión del uso del Xenical se ha visto facilitada por el diseño de terapias curativas que desde la disciplina médica se han elaborado para tratar a las anoréxicas. Un ejemplo de especial interés a este respecto lo constituyen los "programas de paciente expertos" implementados en el Reino Unido. Se trata de terapias pensadas originalmente como espacios horizontales de trabajo de grupo, lideradas por un "paciente experto" ya sanado que comparte con otros pacientes su "experticia" en el tratamiento de su enfermedad, y en particular en el uso de medicamentos como el Xenical. Estos programas, sin embargo, en ocasiones han dado lugar a comunidades de pacientes que se convocan a foros o chat en las que se comparte nuevos usos o usos modificados de los fármacos recetados. Así estos programas de pacientes expertos han resultado ser los principales facilitadores de nuevas formas de resistencia ${ }^{6}$.

Pero ¿de qué tipo de resistencia hablamos? Las comunidades proana adoptan el nombre aportado por el dispositivo médico para las personas que clasifican dentro de dicha condición: anoréxicas. Sin embargo, inmediatamente buscan subvertir su significado patologizado, y lo hacen agregando el prefijo pro- (ana). De tal forma que se trata de una comunidad que acepta para sí una denominación heterónoma, pero que subvierte su significado ("somos anoréxicas, pero eso no es una patología"). Es por ello que podemos hablar de un tipo de resistencia (desujeción) por resignificación. En efecto, el tipo de operación que realizan los movimientos proana es políticamente interesante toda vez que no es la mera negación de un insulto como es el caso de los movimientos queer o negros, dado que "anoréxica" no es un insulto, sino que lo que ocurre es una operación de resituación de significados preestablecidos (Halperin, 2003, pp. 339-343). El tipo de resistencia que las comunidades proana realizan es en contra del dispositivo médico que precluye una forma de vida (la vida anoréxica). El uso modificado del Xenical y compartido comunitariamente en dicha práctica de desujeción no es adjetivo, pues permite hablar de una biopolítica molecular (o más precisamente, de una resistencia desde lo molecular). En efecto, la resignificación sólo se hace posible si el fármaco opera

\footnotetext{
${ }^{6}$ El caso del sistema de expert-patient es tratado en profundidad por Wilson, Patricia (2001). Y específicamente respecto del caso de la anorexia y los movimientos proana por Fox, Nick; Ward, Katie; O'Rourke, Alan (2005).
}

AlPHA No 50 (Julio 2020) PÁGs. 9-20. ISSN 07 16-4254| 15 
y logra estabilizar una condición que de seguir su curso "normal" haría probablemente inviable el vivir como anoréxica y menos formar una comunidad proana.

Ser proana significa, entonces, hacerse de una propia identidad, resignificando un nombre impuesto por el poder médico, pero a su vez conformando con las que son iguales una comunidad.

\section{TESTO YONQUI: HACIA UNA RESISTENCIA POR EXPERIMENTACIÓN}

La resistencia molecular presenta un nuevo ángulo cuando se le observa más allá de un marco de significación/resignificación predeterminado, como el que rige las prácticas de las comunidades proana. Esa es la experiencia que ofrece el trabajo de Beatriz Preciado, quien inicia una experimentación molecular abierta documentada en su obra Testo yonqui, tras ello terminaría transformándose en el filósofo Paul B. Preciado. Tras conseguir de manera legal altas dosis de Testogel (testosterona en gel), comienza a consumirlas con el fin de alterar su constitución biológica, alejándose de la feminidad científicamente establecida y buscando transgredir el género. El marco inicial de la experimentación es similar al que rige el uso del Xenical por parte de las comunidades proana. Preciado se autoadministra un fármaco -cuyo uso normal mirado desde el dispositivo médico es controlar desequilibrios hormonales en las mujeres- buscando explícitamente subvertir el estándar que el medicamento persigue restaurar. En tal sentido, la subversión de los fármacos posibilitará un primer tipo de resistencia dirigido contra el uso normativizado proveído por estos dispositivos farmacológicos y consecuencialmente en contra de las figuras de normalidad que ellos presuponen (el cuerpo sano). Sin embargo, la experiencia documentada por Preciado comienza a partir de ahí a distanciarse de las formas de resistencias seguidas por las comunidades proana. Ya no se trata de recuperar una inscripción simbólica patologizada (como en el caso de la condición médica anoréxica), resignificándola como una forma de vida posible (estabilizada en sus riesgos inminentes de muerte) y legítima (que reclama su lugar en una comunidad que la patalogiza). Por el contrario, lo que hace distinta a la experiencia documentada por Preciado es que no hay allí un intento de resignificación, sino una búsqueda por ir más allá de los horizontes de significados, en este caso, fijados por el género. Al autoadministrarse Testogel, Preciado se expone a modificaciones moleculares que siguen una lógica experimental, esto es, cuyos rumbos son inciertos y por tanto sus significaciones y simbolizaciones se mantienen pendientes. La lógica de experimentación da lugar a un tipo de resistencia que se ubica más allá del dispositivo de intervención farmacológico. No hay ya negociaciones con el marco de normalización presupuesto, sino la creación de mundos radicalmente nuevos.

Es preciso notar que esta apertura es tendencialmente indeterminada en el sentido de que sus consecuencias pueden ir desde formas levemente alteradas de vida biológica (post16 | AlPHA No 50 (Julio 2020) PÁGs. 9-20. ISSN 07 16-4254 
humanas) hasta monstruosidades (alteraciones radicales del bios). Hablamos en tal medida de una biopolítica molecular radicalmente afirmativa. Una creación de vida nueva en sus configuraciones moleculares (al menos en parte de ellas) que desplaza a un segundo momento el problema de la significación e inscripción dentro de un marco de representación simbólica. El momento biopolítico molecular presenta así su rasgo más radical: ocurre dentro de un tiempo -el tiempo de la experimentación- en donde la significación es retrasada y resistida. Cuando Preciado se autoadministra Testogel escribe notas que van registrando los cambios fisiológicos que ocurren en su organismo. Sin embargo, son apuntes llenos de significados pertenecientes a un marco de referencialidad en que el experimento se origina (en su caso, del género femenino) que siempre tiene un retardo respecto de las significaciones finales que se terminarán adoptando. El caso de Preciado es un testimonio de esta textura abierta propia de la experimentación. Primero deja atrás su nombre "Beatriz" para adoptar el de "Paul", lo que testimonia un primer momento de resistencia en donde el género aún sigue orientando las subjetividades, ahora de manera invertida. Sin embargo, los efectos moleculares del Testogel parecen incluso subvertir el propio nombre de Paul, hacerlo incómodo como receptáculo de significación de un bios modificado que busca nuevos significantes para expresarse, para nacer a la vida, más allá de Beatriz-Paul, más allá del género.

El propio Preciado articula teóricamente esta incomodidad al acuñar la tesis de que la revolución que viene será protagonizada por luchas y alianzas somapolíticas (Paul Preciado, 2015, en línea). Las luchas somapolíticas serían luchas revolucionarias en el sentido más estricto del término. No apuntan solo a las estructuras sociales y económicas, sino, y fundamentalmente, a las estructuras que rigen la vida. Es una revolución total como señala Preciado, pero no en términos de lucha por una nueva identidad (política, sexual, o de género) de un sujeto autónomo, sino en cuanto posibilidad de establecer "una multiplicidad de estrategias de resistencias a la normalización" de sujetos dependientes, frágiles y fundamentalmente relacionales. En dicho marco, la experimentación biomolecular adquiere un sentido transindividualizante, pues abre posibilidades nuevas de relacionamiento de vidas que consideradas en su singularidad actual son estériles. La comunidad que se inaugura ya no está fundada en lo que se tiene en común (una identidad, dada o por construir), sino en las relaciones - en su dinámica para ser exactode diversas formas de vida -muchas de ellas sin significación precisa- que comparten un habitad. El habitad no sería sin embargo asimilable a una sociedad política, tiene un estatus más frágil. Aparece para dar cuenta de que lo que hace visible las prácticas de biopolítica (o resistencia) molecular es solo un territorio difuso y prepolítico. El optimismo declamatorio de Preciado (una "nueva revolución total") requiere entonces tomarse con precaución. Si algo inaugura la resistencia molecular en su modalidad de experimentación es un paisaje diverso, pero radicalmente indeterminado incluso en sus lógicas societales más básicas. Tómese por ejemplo el caso de los organismos mutados 
genéticamente a consecuencia de la experimentación molecular, ¿podemos hablar de formas de vida que traen consigo inmediatamente la pretensión de reconocimiento propia de los "derechos humanos"? o ¿estamos más bien en una encrucijada en donde lo primero a constatar es que las fronteras siempre artificiales de lo humano aparecen ahora completamente insuficientes en su pretensión determinadora y protectora de lo que "somos"? Quizás la metáfora ficcional más cercana del habitad que da lugar la resistencia molecular es la Cantina de Mos Eisley de la saga Star Wars: un lugar fascinante en su diversidad, pero mortalmente frágil en su ethos societal. Si es cierto que viene una revolución total, como lo indican las expresiones más lúcidas de la \#OlaFeminista, las luchas a desplegar pueden estar más cerca de la vivencia que del reconocimiento, y en tal sentido apuesta por establecer las condiciones de posibilidad de lo político, o si se prefiere, por alentar la politicidad de formas de vidas diversas.

\section{CONCLUSIONES}

Lo que aquí hemos presentado es una propuesta para pensar las prácticas de resistencia como de sujeción, esto es, como constitutivas de un sí mismo que en su despliegue original da lugar a una vida radicalmente nueva. La condición de posibilidad de esta propuesta está curiosamente asentada en un dispositivo clásicamente regularizador como lo es la práctica o poder farmacológico, fenómeno conocido en la literatura como la "farmaceuticalización de la vida cotidiana" (Camargo y Ried, 2016, pp. 86-88). Sin embargo -y paradojalmente habrá que decir- es la subversión del uso normalizador del fármaco (no su abandono) por parte de comunidades proana o por las experimentaciones político-filosóficas como las llevadas a cabo por Preciado, lo que permite dar lugar a esta nueva práctica de desujeción en sus dos modalidades identificadas en este trabajo: como práctica de resignificación y como práctica de experimentación. En otras palabras, en el seno del dispositivo normalizador se anida su propia superación. No se trata sin embargo de un proceso mágico o exotérico. Muy por el contrario, tanto en las experiencias de las comunidades proana como en la experimentaciones de Preciado, es posible advertir un elemento en común, a saber: la configuración de una comunidad de resistentes: de una nueva forma de vida anoréxica en un caso, o de una vida desinscrita de los cánones, pero abrazada a la indeterminación cómplice de los inclasificados (los raros), en el caso de Preciado. En última instancia, la desujeción supone no solo constituciones de un sí mismo singular, sino de un común nuevo resignificado indeterminado. En el caso de las comunidades proana se trata de un común constituido en la subversión de la significación estándar del fármaco. Más aún, lo que permite hacer comunidad es la emergencia de un ethos de vida no reconocida, patologizada en su propio bios (Camargo y Ried, 2017). En Preciado, a su vez, la comunidad es una posibilidad aún más incierta. Se trata siempre del momento posterior a la lógica de experimentación 
molecular desplegada en un escenario de indeterminación radical (la vida resultante puede ser monstruosa), pero en su propia originalidad logra convocar la solidaridad comunitaria de otras vidas monstruosas que yacen en un habitad, aun no reconocidas incluso para sí mismas.

Finalmente, si consideramos a estas prácticas de resistencias como interpeladoras de la vida política, diríamos que si lo que se origina en las dos modalidades de desujeción analizadas son formas de vida indeseables, su primera lucha es por existir como formas de vida válidas, y en tal medida por ampliar los contornos de lo político. Una lucha desplegada frente a una sociedad política habitualmente hostil, cuya respuesta primaria esperable es la inmunización, la exclusión y el exterminio. Hablamos por tanto de una lucha bioexistencial.

Este artículo es resultado del proyecto FONDECYT N¹170940

\section{OBRAS CITADAS}

Camargo, Ricardo (2010). "Población y pueblo: notas acerca de la posibilidad del cambio emancipador en Michel Foucault". En: LEMM, Vanessa. Michel Foucault: neoliberalismo y biopolítica. Santiago: UDP.

Camargo, Ricardo y Ried, Nicolás (2017). "Biopolítica y Verdad: el caso de Marchiafava-Bignami en el dispositivo judicial chileno". Revista Chilena de Derecho y Tecnología. V. 6, No 2: 121-133.

_ (2016). "Towards a genealogy of pharmacological practice". Medicine, Health Care and Philosophy. V. 19, Nº1: 85-94.

Castro, Edgardo (2004). El vocabulario de Michel Foucault. Un recorrido alfabético por los temas, conceptos y autores. Buenos Aires: Universidad Nacional de Quilmes.

Foucault, Michel (2010). "Michel Foucault, una entrevista: sexo, poder y política de la identidad". En su: Obras esenciales. Madrid: Paidós.

- (2008). Historia de la sexualidad. Tomo 1: La voluntad del saber. Buenos Aires: Siglo XXI editores.

— (1995). “¿Qué es la crítica?”. En: $\Delta \alpha i ́ \mu \omega v$, revista de filosofía. № 11.

Fox, Nick; Ward, Katie y O'Rourke, Alan (2005). "Pro-anorexia, weight-loss and the internet: an 'anti-recovery' explanatory model of anorexia". En: Sociology of Health \& Illness.

Goolding, Sigal (2008). "Being anorexic: hunger, subjectivity, and embodied morality". En: Medical anthropology quarterly.

Halperin, David (2003). "The normalization of queer theory". En: Journal of homosexuality. Haworth Press. 
Heller, Kevin Jon (1996). "Power, subjectivation and resistance in Foucault". En: SubStance, $\mathrm{N}^{\mathrm{o}} 1 \mathrm{~V} .25$.

Hollander, Jocelyn A. y Einwohner, Rachel L. (2004). “Conceptualizing resistance”. En: Sociological forum, V. 19, $\mathrm{N}^{\circ} 4$.

Hope, Tony; Tan, Jacinta; Stewart, Anne y Fitzpatrick, Ray (2011). "Anorexia nervosa and the language of authenticity". En: Hasting center report. V. 41, № 6 .

Karmy, Rodrigo; Yuing, Tuillang (Eds.) (2014). Biopolíticas, gobierno y salud pública. Miradas para un diagnóstico diferencial. Santiago: Ocho libros.

Metzl, Jonathan; Kirkland, Anna (Eds.) (2010). Against health. How health became the new morality. New York: NYU press.

Nadesan, Majia Holmer (2010). Governmentality, Biopower, and Every Day Life. London: Taylor y Francis.

O’Connor, Richard y Van Esterik, Penny (2008). "De-Medicalizing anorexia: a new cultural brokering". En: Royal anthropological institute of Great Britain and Ireland.

Pickett, Brent L. (1996). “Foucault and the politics of resistance". En: Polity 28 N$^{\circ}$ 4: 445-466.

Preciado, Beatriz (2014). Testo yonqui. Sexo, drogas y biopolítica. Barcelona, España: Paidós.

Preciado, Paul Beatriz (2015). "La revolución que viene: Luchas y alianzas somatopolíticas". Conferencia del sábado 6 de junio de 2015 en el Museo de Arte Latinoamericano de Buenos Aires (MALBA). Disponible en línea: https://www.youtube.com/watch?v=vsV2e_FBreA

Rancière, Jacques (2006). "Política, identificación, subjetivación". En su: Política, policía, democracia. Santiago: LOM.

Ried, Nicolás (2016). Los momentos de la resistencia. Santiago: Universidad de Chile. Tesis de grado.

Rose, Nikolas y Miller, Peter (2008). Governing the present. Administering economic, social and life. Cambridge: Polity Press.

Wilson, Patricia (2001). "A policy analysis of the Expert Patient in the UK: self-care as an expression of pastoral power?". Health and social care in community 9: 134-142. 